\title{
ESPECTROS DETERMINÍSTICOS Y PROBABILÍSTICOS PARA LA EVALUACIÓN DEL PELIGRO SÍSMICO EN ESTRUCTURAS DE LA REGIÓN DE TACNA
}

\author{
DETERMINISTIC AND PROBABILISTIC SPECTRUMS FOR THE EVALUATION OF \\ SEISMIC HAZARD IN STRUCTURES OF THE TACNA REGION
}

Ever Rudy Ancco Huanacuni ${ }^{1}$ Edgar Hipolito Chaparro Quispe ${ }^{2}$

Información del artículo: Recibido: $11 / 05 / 2019$ Aceptado: $26 / 11 / 2020$

\footnotetext{
${ }^{1}$ Ingeniero civil. E-mail: ever.ancco.huanacuni@gmail.com, ${ }^{2}$ Docente en la Facultad de Ingeniería, Universidad Privada de Tacna. E-mail: edgar.hcq@gmail.com
} 


\section{Resumen}

El estudio tiene la finalidad de brindar criterios técnicos para determinar el peligro sísmico en la región de Tacna mediante espectros elásticos determinísticos y probabilísticos que, en consecuencia, puedan representar la amenaza sísmica relacionada a dicha región. Este estudio ha considerado los sismos que han sido registrados instrumentalmente desde enero de 1965 hasta el 22 de mayo del 2018 (comprendidos en el sur del Perú y norte de Chile). Siendo la principal fuente de información los catálogos sísmicos emitidos por el Servicio Geológico de los Estados Unidos (USGS). Los resultados reflejan la atenuación de los eventos sísmicos de una determinada magnitud a una determinada distancia, en base a un análisis determinístico y/o probabilístico, en cuyo caso este último es extrapolado a una determinada probabilidad anual de excedencia. Estableciendo de esta forma mapas de peligro sísmico en ordenadas espectrales.

Palabras clave: Atenuación, Determinístico; Espectro; Magnitud; Probabilístico; Sismo.

\section{Abstract}

The present study has the purpose of providing technical criteria to determine the seismic hazard in the Tacna region through deterministic and probabilistic elastic spectra that, consequently, may represent the seismic threat related to said region. This study considered earthquakes have been instrumentally recorded from 1965 to May 22, 2018, comprised in southern Peru and northern Chile. The main source of information is the seismic catalogs issued by the United States Geological Survey (USGS). The results reflect how it is the attenuation of these seismic events of a certain magnitude at a certain distance, based on a deterministic and / or probabilistic analysis, in which case the latter is extrapolated to a certain annual probability of exceedance. Establishing seismic hazard maps in spectral ordinates in this way.

Keywords: Attenuation, Deterministic; Spectrum; Magnitude; Probabilistic; Earthquake. 


\section{Introducción}

La Región de Tacna que se encuentra ubicado el extremo sur occidental del Perú, entre las coordenadas geográficas $16^{\circ} 18^{\prime}$ y $18^{\circ} 20^{\prime}$ latitud sur y $69^{\circ} 28^{\prime}$ y $71^{\circ} 02^{\prime}$ de longitud oeste.

Los eventos sísmicos de gran intensidad ocurridos en esta región, han ocasionado la pérdida de vidas humanas, daños materiales y pérdidas económicas. Bajo este concepto la confiabilidad estructural proporciona un campo de estudio amplio para evaluar la importancia de las solicitaciones que se aplican a los modelos matemáticos en el análisis de estructuras. Siendo los sismos en potencia, la principal amenaza de dicha Región.

Como consecuencia de lo anterior, el análisis sísmico de las estructuras en la región de Tacna, es quizás la más importante, porque de ella depende el éxito del diseño. Es la parte creativa de la ingeniería y en ella se decide el desempeño de una estructura en función a sus cualidades, en tal sentido la determinación del peligro sísmico mediante la aplicación de espectros determinísticos y/o probabilísticos permite una representación de la amenaza sísmica relacionada a dicha región para cada periodo estructural.

Según el reglamento nacional de edificaciones en la norma E.030 "Diseño Sismo resistente", se desprenden en el Capítulo 2 de dicha norma los estudios de peligrosidad sísmica. En ella, el Perú se divide en cuatro zonas sísmicas, desde la Z1 hasta la Z4 y PGAs desde $0.10 \mathrm{~g}$ hasta $0.45 \mathrm{~g}$, respectivamente, las cuales sintetizan el peligro sísmico del Perú. Por otro lado, dicha norma ha tenido importantes actualizaciones en los años 2016 y 2018 lo que indica el desarrollo ingenieril en esta línea de investigación. Además, de la necesidad de nuevas metodologías e investigaciones respecto de este tema. Este es uno de ellos, que espera ser un aporte y al que otros puedan sumarse a la tarea de investigar, para ofrecer otras alternativas y/o técnicas que contribuyan al desarrollo científico, que puedan ser evaluados en beneficio de la sociedad.

\section{Objetivos}

Determinar los espectros determinísticos y probabilísticos para la evaluación del peligro sísmico en estructuras de la región de Tacna.

\section{Metodología}

Los métodos utilizados para evaluar la peligrosidad sísmica pueden ser determinísticas y probabilísticas. Ambos consideran la sismicidad como un fenómeno estacionario, y suponen que los sismos que ocurrirán en el futuro serán de las mismas características que los sucedidos en el pasado.

Los métodos deterministas analizan la sismicidad de cada zona sismogénica que afecta al lugar cuya peligrosidad se estudia, con el objeto de estimar el máximo sismo potencial (se considera un único terremoto para el análisis y se acepta que volverá a repetirse en el futuro). Este máximo terremoto potencial, o razonable esperable, se sitúa en el lugar de cada zona sismogénica más próximo al emplazamiento, punto al que, mediante una ley de atenuación, se traslada el parámetro de velocidad, aceleración, etc. que expresa el efecto del terremoto.

La peligrosidad final se toma como el máximo de las acciones correspondientes a todas las zonas sismogénicas. Si estas no han sido definidas, el proceso se realiza considerando directamente los efectos de cada terremoto de la región que rodea al emplazamiento (Véase la figura 1.) 


\section{Figura 1}

Proceso para el análisis del peligro sísmico determinístico

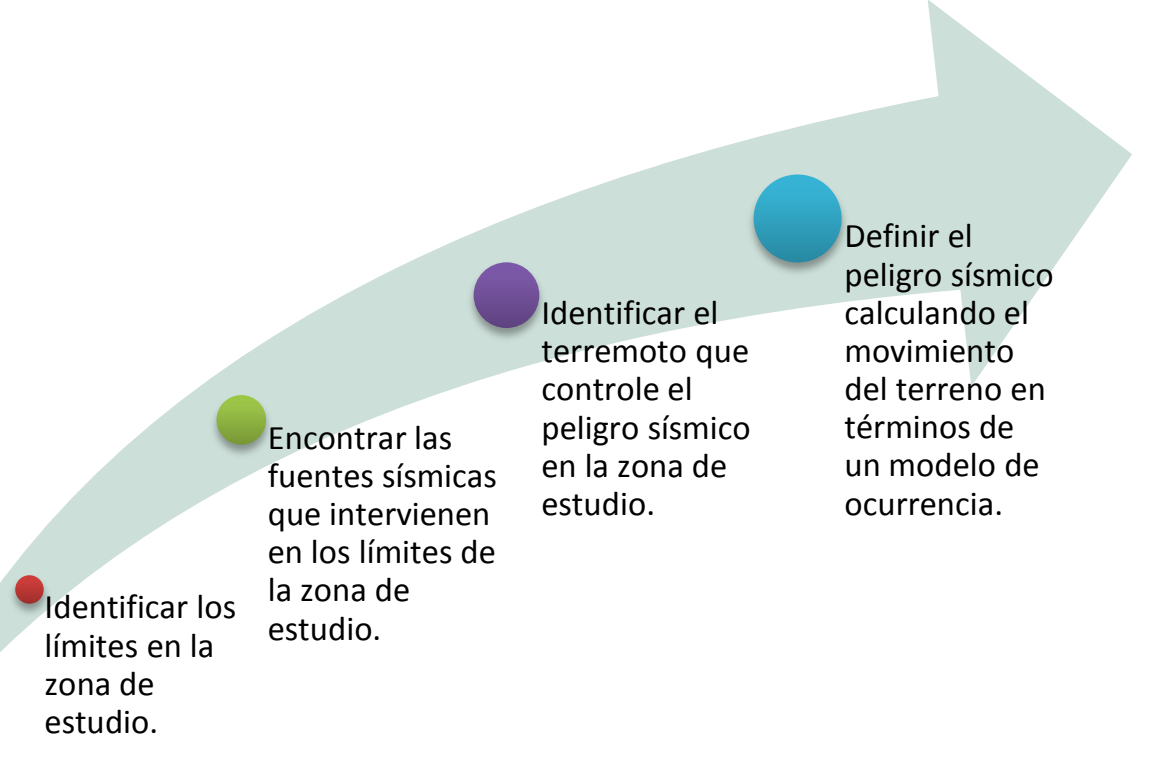

Nota: Adaptado de Kramer (1996).

\subsection{Peligro Sísmico Probabilístico}

Los métodos probabilistas parten del conocimiento de la sismicidad pasada para deducir las leyes estadísticas que regirán la actividad sísmica futura. Con ello se estima la probabilidad de que los distintos niveles de movimiento del suelo sean superados en un plazo dado. En este caso, una vez determinada las zonas sismogénicas, la sismicidad de cada una de ellas se expresa con una ley de ocurrencia de la forma Gutenberg - Richter. Así cada zona queda caracterizada por los parámetros $a, b$ y las magnitudes máxima y mínima que acotan la línea de ajuste. Igual que en los estudios deterministas, una ley de atenuación adecuada traslada la influencia de cada zona al punto de análisis y la peligrosidad se evalúa sumando la influencia de cada zona al punto de análisis y la peligrosidad se evalúa sumando la influencia de todas las fuentes consideradas.

A diferencia de los deterministas, que indican la probabilidad del valor máximo, estos métodos proporcionan la probabilidad correspondiente a supuestos muy variados. De esta manera es posible elegir el valor que responde al tipo de estructura que se quiere diseñar. Esta ventaja, unida al hecho sismológicamente significativo de no basarse en un valor máximo sino en consideraciones estadísticas, (cuya validez quedará limitada por la extensión y homogeneidad de la muestra de terremotos, y su capacidad de incluir todos los eventos más importantes), ha motivado que los métodos probabilistas sean hoy mucho más empleados que los deterministas. Por ello es objeto de un análisis más extenso (Véase la figura 2). 


\section{Figura 2}

Proceso para el análisis del peligro sísmico probabilístico

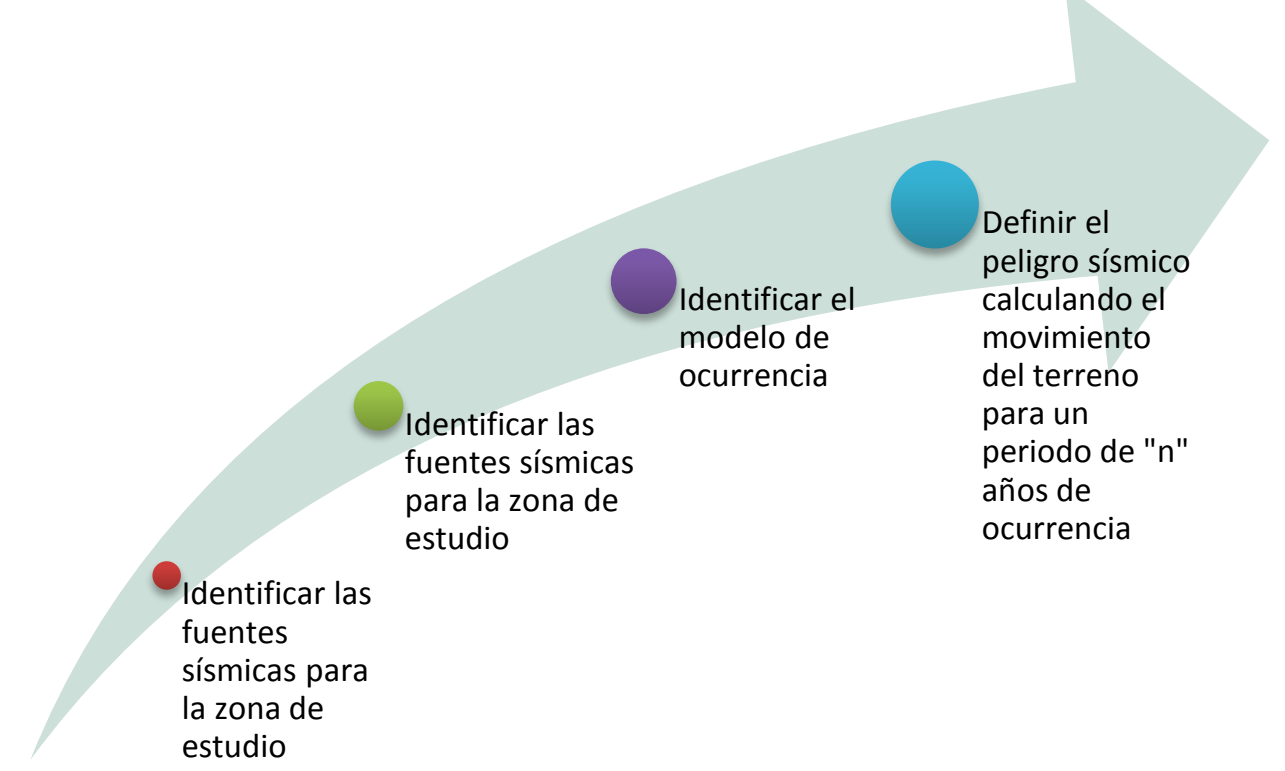

Nota: Adaptado de Kramer (1996).

La región de Tacna está situada al sur del Perú perteneciente al desierto costero peruano que limita al norte con los departamentos de Moquegua y Puno; por el este con Bolivia y Chile; por el oeste con el océano pacifico y por el sur con Chile, cuenta con cinco zonas sismogénicas actualizadas y estudiadas además de un catálogo con 4442 registros de eventos sísmicos (instrumentados de 1965 a 2018) para el sur del Perú y norte de Chile.

El catalogo sísmico que brinda el Servicio Geológico de los Estados Unidos (USGS) posee eventos sísmicos con magnitudes de: $\mathrm{Mw}, \mathrm{Mb}, \mathrm{Ms}$ y en algunos casos, $\mathrm{Ml}$ por lo que fue necesario homogenizar la base de datos para realizar posteriores análisis.

Para el procesamiento de datos se eligió la magnitud momento $\mathrm{Mw}$ como la escala base. Debido a que es la única escala de magnitud que no se satura. Además, las relaciones de atenuación usadas actualmente para el movimiento del suelo y las ordenadas espectrales están basadas en Mw.

Estas divisiones sismológicas se muestran en la Figura 3, Figura 4 y Figura 5. Pertenecientes a la clasificación de fuentes de interfase, interplaca y superficiales según SENCICO. (2016), esta clasificación da lugar las siguientes fuentes sismogénicas: a) F5, b) F11, c) F12, d) F19 y f) F24. 
Figura 3

Eventos sísmicos en la fuente sismogénica de Interfase (F5) para la Región de Tacna

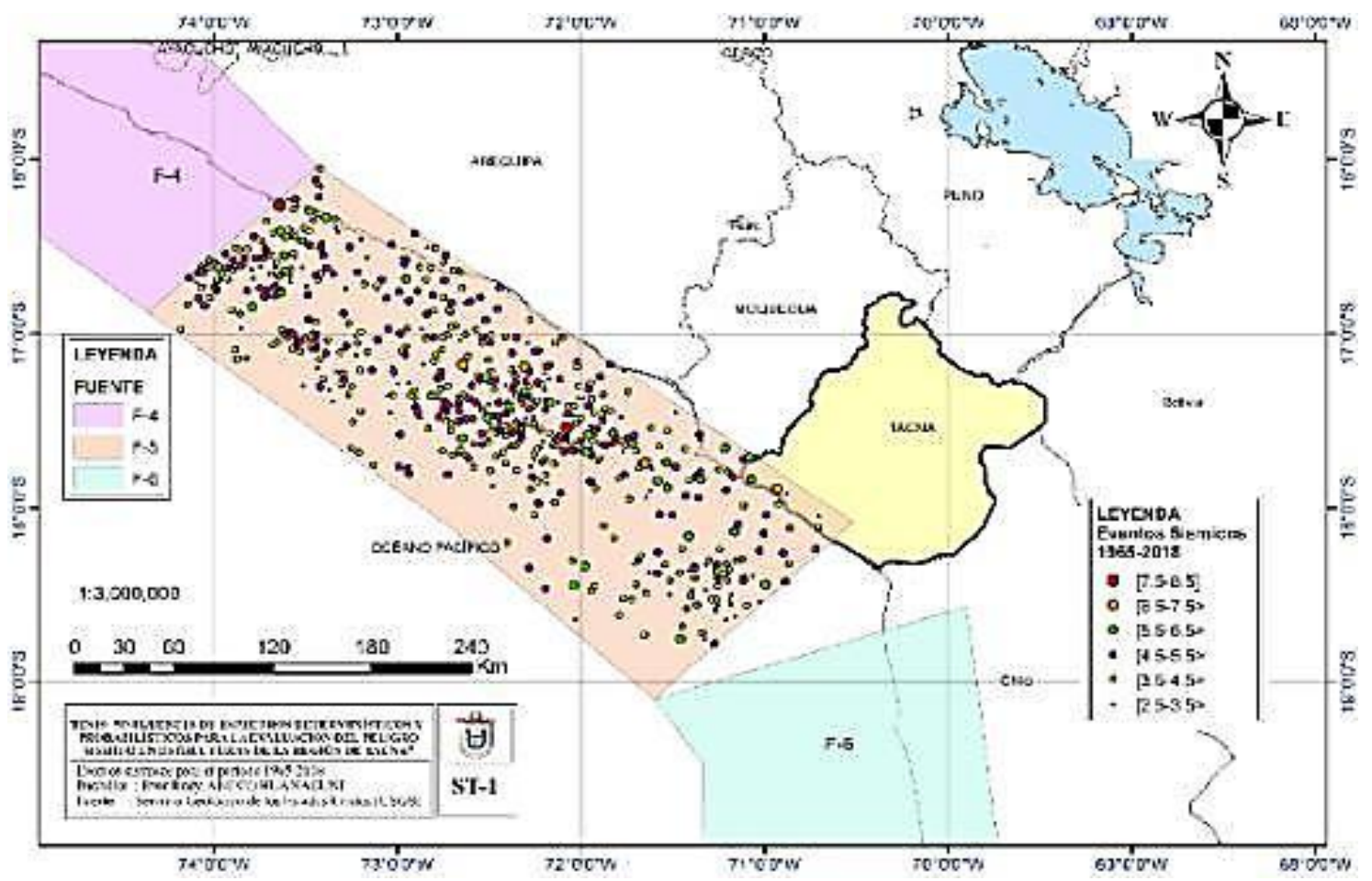

Nota: diagramación del autor.

\section{Figura 4}

Eventos sísmicos en las fuentes sismogénicas de Interplaca (F11, F12, F19) para la Región de Tacna

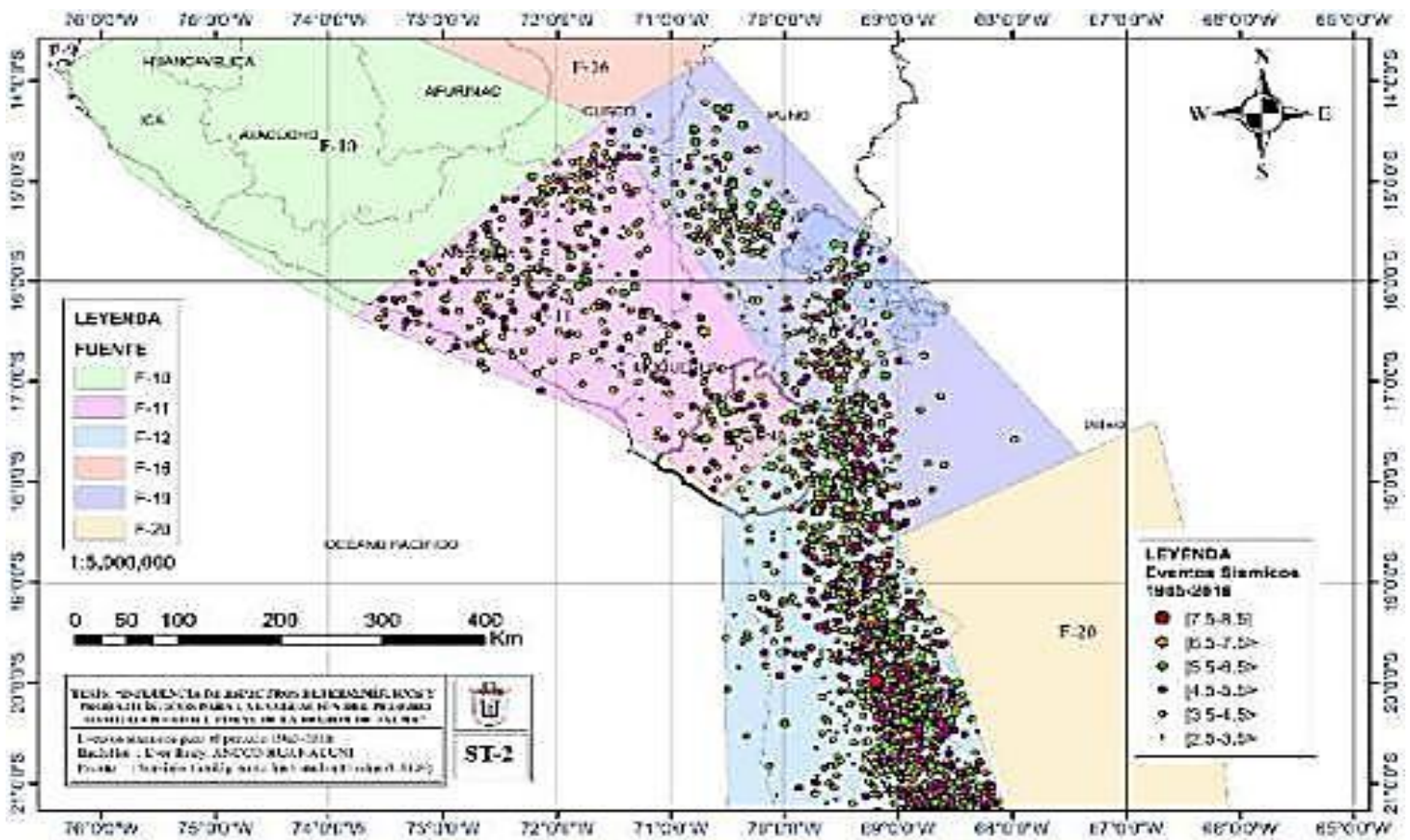

Nota: Diagramación del autor. 


\section{Figura 4}

Eventos sísmicos en la fuente sismogénica superficial (F24) para la Región de Tacna

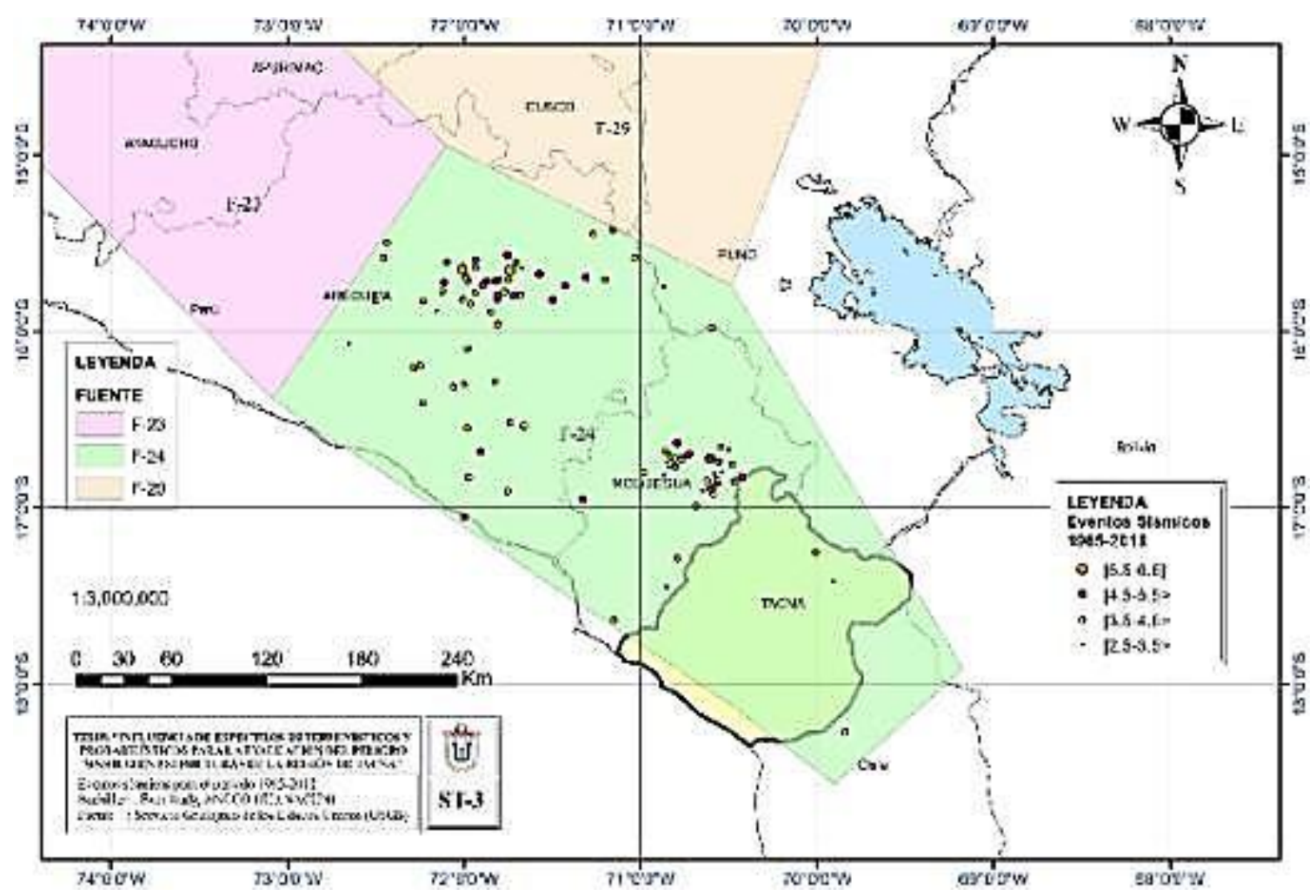

Nota. Diagramación del autor.

Figura 5.

Recurrencia sísmica para la Región de Tacna

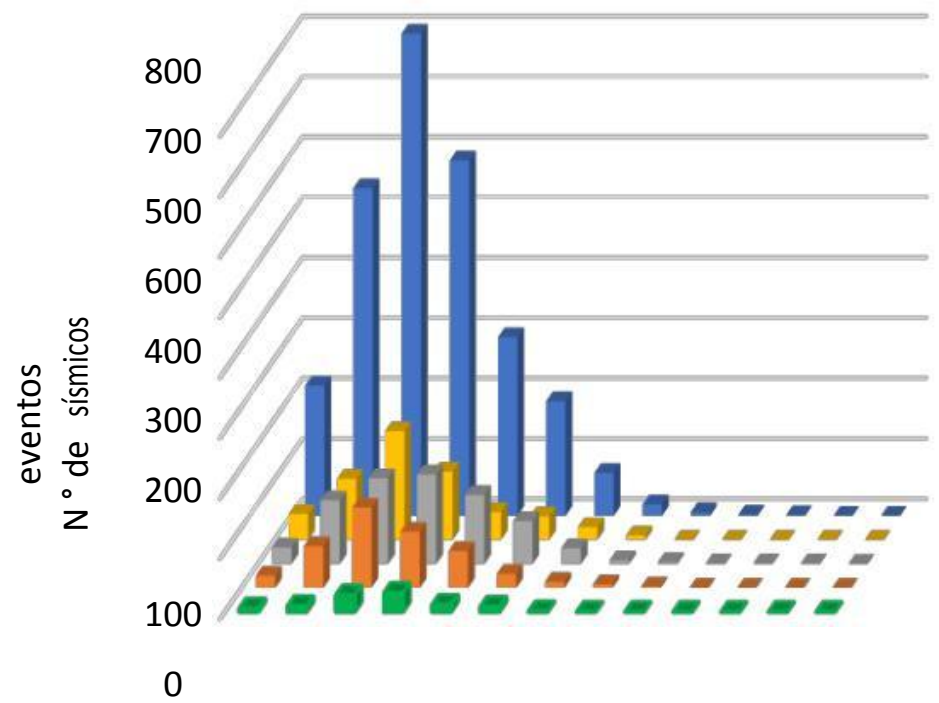

Magnitud (Mw)

m-24 $\approx \mathrm{F}-11 \backsim \mathrm{F}-05 \backsim \mathrm{F}-19 \backsim \mathrm{F}-12$

Nota. Diagramación del autor. 
Leyes de Atenuación de Youngs, Chiou, Silva, \& Humphrey (1997) define las características de sitio en una clasificación de roca y suelo profundo, utilizando la magnitud de momento Mw para la medida de cada evento:

\section{Sismos por Subducción en Roca}

La relación de atenuación para la aceleración espectral de respuesta horizontal ( $5 \%$ amortiguado) en roca está dada por la siguiente función:

$$
\begin{gathered}
\operatorname{Ln}(y)=0.2418+1.414 \mathrm{M}+\mathrm{C} 1+\mathrm{C} 2(10-\mathrm{M})^{\wedge} 3+\mathrm{C} 3 \\
\operatorname{Ln}\left(\text { rrup }+1.7818 \mathrm{e}^{\wedge} 0.554 \mathrm{M}\right)+0.00607 \mathrm{H}+0.3846 Z \mathrm{~T}
\end{gathered}
$$

\subsection{Sismos por Subducción En Suelo}

La relación de atenuación para la aceleración espectral de respuesta horizontal ( $5 \%$ amortiguado) en suelo está dada por la siguiente función:

$$
\begin{aligned}
& \operatorname{Ln}(y)=-0.6687+1.438 \mathrm{M}+\mathrm{C} 1+C 2(10-\mathrm{M})^{\wedge} 3+\mathrm{C} 3 \\
& \operatorname{Ln}\left(\mathrm{R}+1.097 \mathrm{e}^{\wedge} 0.617 \mathrm{M}\right)+0.00648 \mathrm{H}+0.3643 \mathrm{ZT}
\end{aligned}
$$

En donde para ambos casos:

$\mathrm{Y}=$ aceleración espectral en (g). $\mathrm{M}=$ magnitud momento ( $\mathrm{Mw})$.

rup $=$ distancia más cercana a la rotura $(\mathrm{Km}) . \mathrm{H}=$ profundidad $(\mathrm{Km})$.

$\mathrm{ZT}$ = tipo de fuente, 0 para Interfase, 1 para intraplaca

\subsection{Sadigh, Chang, Egan, Makdisi, \& Youngs (1997)}

Esta relación está basada principalmente en sismos de la Costa Oeste de los Estados Unidos para los sismos continentales.

$$
\operatorname{Ln}(\mathrm{Sa})=\mathrm{C} 1+\mathrm{C} 2 \mathrm{M}+\mathrm{C} 3(8.5 \mathrm{M})^{\wedge} 2.5+\mathrm{C} 4(\operatorname{Ln}(\mathrm{R}+\exp (\mathrm{C} 5+\mathrm{C} 6 \mathrm{M})))+\mathrm{C} 7 \mathrm{Ln}(\mathrm{R}+2)
$$

Donde:

Sa $=$ aceleración espectral en $(\mathrm{g})$

$\mathrm{M}=$ magnitud momento $(\mathrm{Mw})$

$\mathrm{R}=$ distancia más cercana a la rotura $(\mathrm{Km})$ 


\subsection{Completitud de la Muestra Sísmica}

La incompletitud de las muestras de eventos acontecidos hace que no encajen en la ecuación planteada para la relación de recurrencia de Gutenberg y Richter. Es por ello que en este estudio se realizó la completitud usando la metodología planteada por Steep.

El procedimiento consiste en agrupar los eventos en clases de magnitudes y cada clase se modela como un proceso de punto en el tiempo. La secuencia de los eventos puede ser modelada por una distribución de Poisson. Se considera que la muestra es estacionaria si la media, la variancia y otros parámetros se mantienen en cada intervalo de tiempo (o en el periodo de ventana evaluado).

\subsection{Relación Gutenberg-Richter}

Es la determinación que en un rango de magnitudes la relación del logaritmo de la frecuencia (acumulada de eventos) con la magnitud, es lineal. El inicio de dicho rango será la magnitud mínima, y el final es la magnitud máxima.

$$
\log N=a-b M
$$

Donde, es la frecuencia acumulada de eventos que se tienen registrados mayores o iguales a una magnitud, es la magnitud, y son los parámetros de la ecuación lineal que se obtiene mediante mínimos cuadrados

\section{Resultados}

Los aspectos relevantes a lo largo del estudio fueron: Peligro sísmico determinístico, Peligro sísmico probabilístico y mapas de isoaceleraciones. En cuanto se refiere al análisis determinístico se ha seccionado al distrito de Pocollay como lugar de estudio el cual se encuentra ubicado entre las coordenadas $17^{\circ} 50^{\prime} 39.84^{\prime \prime}$ latitud sur y $70^{\circ} 13^{\prime} 6.6^{\prime \prime}$ longitud oeste, perteneciente a la fuente sismogénica F12 en la que prevalecen eventos sísmicos de intraplaca. En cuanto se refiere al análisis probabilístico se han evaluado 4442 sismos registrados en la zona de estudio en cinco fuentes sismogénicas mediante las leyes de atenuación expresadas en las ecuaciones: (1), (2) y (3). Logrando generar además mapas de isoaceleraciones.

\subsection{Peligro sísmico determinístico:}

El sismo a evaluar es del 25 de febrero de 1983 con una magnitud de $6.9 \mathrm{Mw}$ e hipocentro ubicado a $18.27^{\circ}$ latitud sur y $69.44^{\circ}$ longitud oeste, a $146 \mathrm{~km}$ de profundidad y una distancia a la ruptura de $105.946 \mathrm{~km}$., los resultados se aprecien en la figura 6:

\subsection{Peligro sísmico probabilístico}

Los resultados se obtuvieron con la aplicación del programa Crisis 2015, en la que se incluyen aceleraciones horizontales para periodos de $\mathrm{T}=0.0 \mathrm{~s}$ a $\mathrm{T}=3 \mathrm{~s}$, con un incremento de 0.05 segundos correspondientes a periodos de retorno de 43, 72, 475, 975 y 4975 años con 5\% de amortiguamiento en 23 distritos de la región de Tacna. En la Tabla siguiente se aprecia los datos de ingreso al programa ya mencionado 


\section{Figura 6}

Espectros sísmicos determinísticos para la ley de atenuación de Youngs en roca y suelo

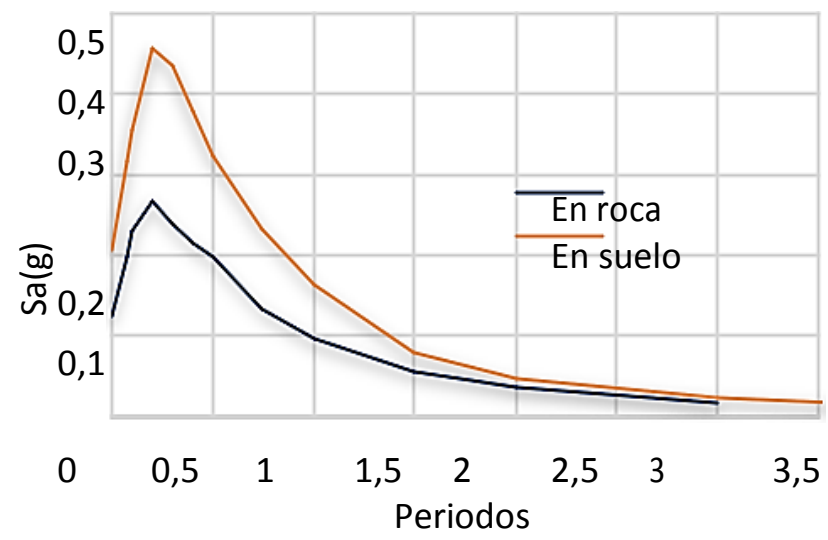

Nota. Diagramación del autor.

Tabla 1

Resumen de los valores de la relación Gutenberg Richter

\begin{tabular}{lccccc}
\hline \multicolumn{1}{c}{ Parámetros } & Interfase & \multicolumn{3}{c}{ Intraplaca } & Superficial \\
\hline \multirow{3}{*}{$(1)$} & F-05 & F-11 & F-12 & F-19 & F-24 \\
b (1) & 3,7 & 3,9 & 6,2 & 3,7 & 4,2 \\
Mo & 0,7 & 0,8 & 1,1 & 0,7 & 1,0 \\
${ }_{\lambda}(2)$ & 4,5 & 4,5 & 4,5 & 4,5 & 4,5 \\
$\sigma(b)$ & 5,8 & 2,6 & 13,3 & 2,5 & 0,6 \\
$\beta=\ln (10) b$ & 0,0 & 0,0 & 0,1 & 0,1 & 0,1 \\
$\sigma(\beta)=\ln (10) \sigma(b)$ & 1,5 & 1,8 & 2,5 & 1,6 & 2,2 \\
$E(\mathrm{Mu})$ & 0,1 & 0,1 & 0,2 & 0,3 & 0,3 \\
$\sigma(\mathrm{Mu})$ & 8,0 & 6,0 & 6,5 & 6,0 & 6,0 \\
$M 1=E(\mathrm{Mu})-\sigma(\mathrm{Mu})$ & 8,0 & 6,0 & 6,5 & 6,0 & 6,0 \\
$\mathrm{M} 1=\mathrm{E}(\mathrm{Mu})+\sigma(\mathrm{Mu})$ & 8,0 & 6,0 & 6,5 & 6,0 & 6,0 \\
\hline
\end{tabular}

Nota. (1) Parámetro de Gutenberg-Ricther. (2) Tasa anual de excedencia de Mo. Diagramación del autor.

\subsection{Mapas de Isoaceleraciones}

El estudio ha permitido elaborar tres mapas de isoaceleraciones para periodos estructurales de $\mathrm{T}=0.0 \mathrm{~s}, \mathrm{~T}=0.20 \mathrm{~s}$ y $\mathrm{T}=1.00 \mathrm{~s}$ con $5 \%$ de amortiguamiento. El primero con un tiempo de retorno de 475 años (equivalente a un $10 \%$ de excedencia en 50 años) y los dos siguientes con un tiempo de retorno de 4975 años (equivalente a un 1\% de excedencia en 50 años). 


\section{Figura 7}

Mapa de aceleraciones máximas del suelo en la región de Tacna para un periodo estructural de $T=0.00 \mathrm{~s}$.
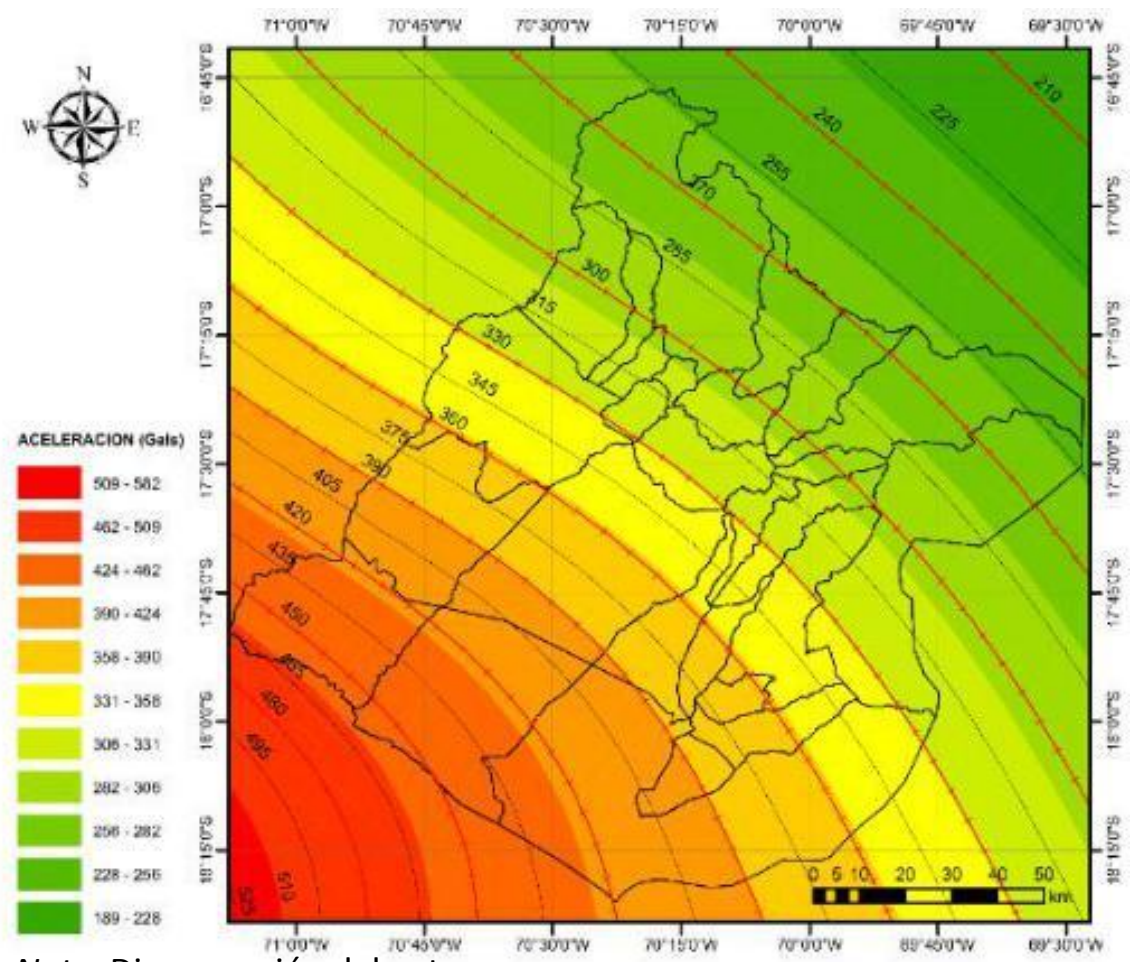

Nota. Diagramación del autor.

\section{Discusión}

El primer espectro normativo (Figura 8) se obtiene para el distrito de Pocollay con una clase de suelo S1 (roca o suelo muy rígido) y el segundo (Figura 9) corresponde a la clase de suelo S2 que es un suelo intermedio. La clase de suelo S1 se usó para la comparativa con el espectro obtenido en roca (según Youngs en roca) y la clase de suelo S2 con el obtenido en suelos (según Youngs en suelos).

Con respecto al espectro determinístico en roca según (Youngs, Chiou, Silva, \& Humphrey, 1997), para el sismo del 25 de febrero de 1983 (6.9 Mw), todos los valores caen por debajo del espectro normativo, pero los espectros obtenidos son bastante parecidos (ver Figura 8 y 9), con la diferencia de la pendiente ascendente en los periodos menores a 0.1 segundos.

Debido a que el espectro de aceleraciones de la norma técnica sismorresistente E-030 es probabilístico, con un $10 \%$ de probabilidad de excedencia en 50 años y un $5 \%$ de amortiguamiento. No es recomendable comparar cuantitativamente los valores de un espectro probabilístico con otro determinístico. Es la razón por la cual se realiza una comparación cualitativa (forma del espectro) y no cuantitativa en los espectros mostrados anteriormente. 


\section{Figura 8}

Comparación del espectro sísmico determinístico según ley de atenuación de Youngs para roca y E-030.

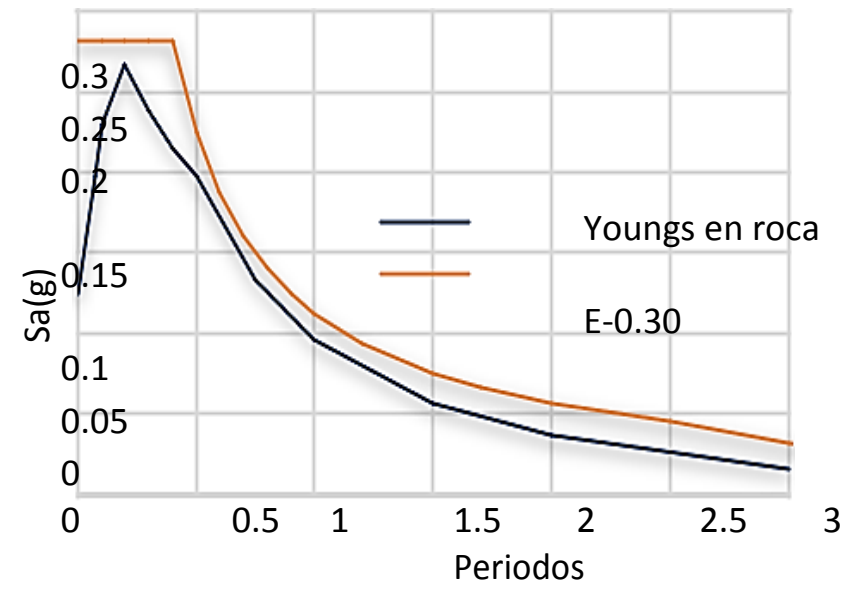

Nota. Diagramación del autor.

\section{Figura 9}

Comparación del Espectro Sísmico Determinístico según

Ley de Atenuación de Youngs para Suelo y E-030

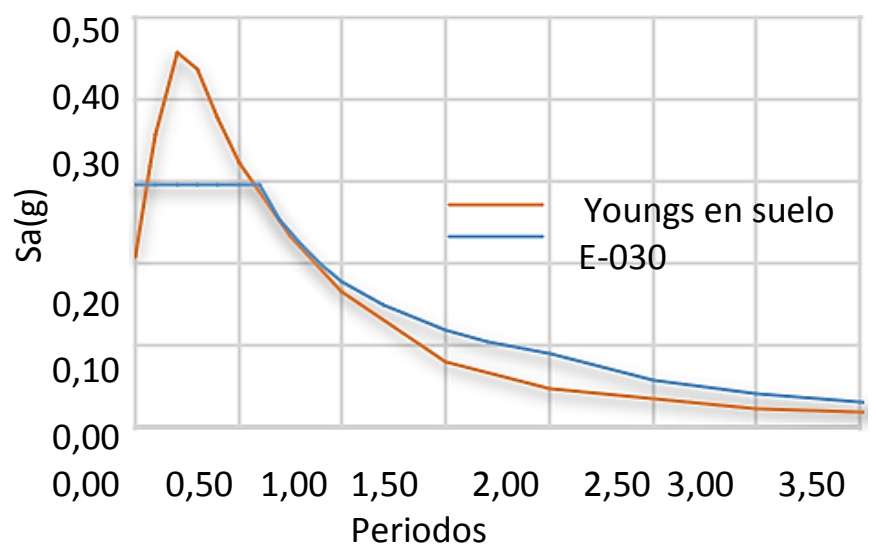

Nota. Diagramación del autor.

Los resultados del estudio de peligrosidad sísmica probabilística obtenido mediante el uso del software Crisis 2015 ver 4.1. (Ver Figura 10) ha servido para la comparación con las normativas E-0.30 y ASCE/SEI 7-10.

En la figura 10 se muestra la forma espectral de cinco aceleraciones de peligro sísmico para el distrito de Pocollay, donde cada ordenada espectral es obtenida de acuerdo a un periodo de excedencia (de 44, 73, 475, 975 y 4975 años) para un 5\% de amortiguamiento con respecto al crítico, teniendo en cuenta la eventualidad de sismos moderados cercanos al sitio y sismos grandes alejados del sitio. 


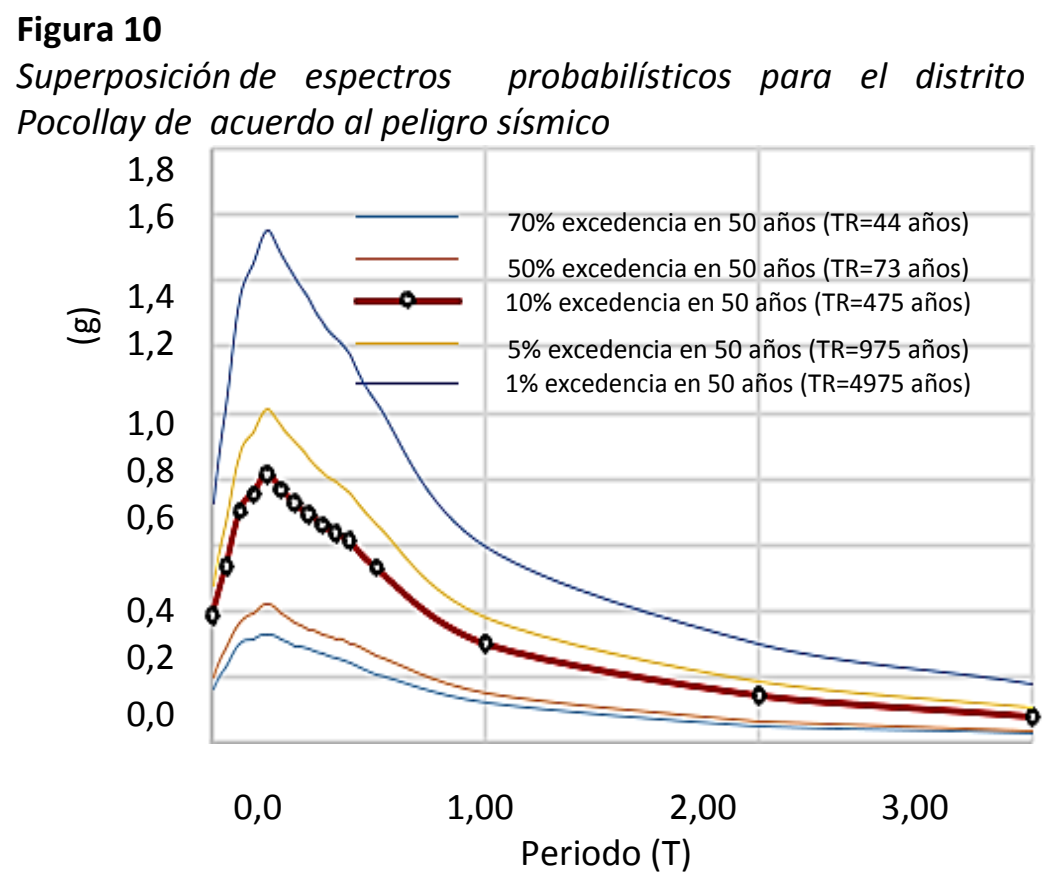

Nota. Diagramación del autor.

En la Figura 11. Se puede apreciar la comparación del espectro calculado probabilísticamente de acuerdo al grado de peligro sísmico en la región de Tacna versus los espectros normativos E-030 y ASCE/SEI 7. Cada uno de ellos escalados adecuadamente para un periodo estructural de 1.0 segundo, que demuestra el gran parecido de la forma espectral (validando de esta forma la investigación realizada), a diferencia de la pendiente ascendente para periodos menores a 0.1 segundos.

\section{Figura 11}

Superposición de espectros de aceleración sísmica para el distrito de Pocollay (escalado)

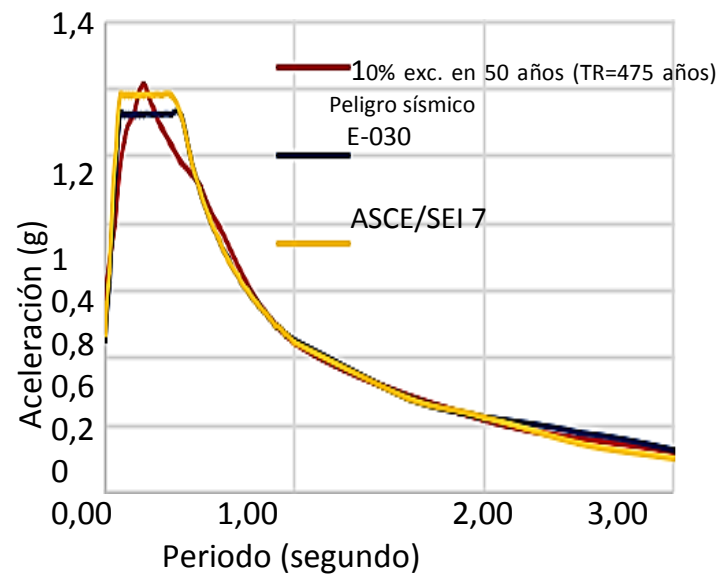

Nota. Diagramación del autor.

\section{Conclusiones}

A manera de conclusiones; la determinación de espectros determinísticos permitió evaluar la ley de atenuación propuesta por (Youngs, Chiou, Silva, \& Humphrey, 1997), estableciendo que, 
cuando se varía la distancia entre el epicentro y la zona de estudio, esta genera una mayor variación en los valores de las aceleraciones espectrales. Por otra parte, la determinación de espectros probabilísticos se pudo establecer mediante uso del software Crisis 2015 Ver 4.1. El cual ha permitido evaluar las leyes de atenuación propuestas por (Youngs, Chiou, Silva, \& Humphrey, 1997) y (Sadigh, Chang, Egan, Makdisi, \& Youngs, 1997) para el cálculo de las aceleraciones espectrales.

Se determinó el Peligro sísmico para 23 distritos de la región de Tacna, de los cuales las máximas aceleraciones horizontales se encuentran en el distrito de Ite.

Para el distrito de Ite (máximas aceleraciones), para los periodos estructurales de $\mathrm{T}=0.0 \mathrm{~s}$, $\mathrm{T}=0.20 \mathrm{~s}$ y $\mathrm{T}=1.00 \mathrm{~s}$ con $5 \%$ de amortiguamiento para una vida útil de 50 años y un periodo de retorno de 475 años. Oscilan alrededor de los $0.47 \mathrm{~g}$ (mayor al normativo en 4\%), 0.99g y $0.36 \mathrm{~g}$ respectivamente. Asi mismo sobre los mapas de isoaceleraciones, se puede afirmar que los valores de PGA obtenidos, son de mejor discretización, en comparación a las cuatro zonas sísmicas mostradas en la N.T.P. E-0.30.

\section{Referencias Bibliográficas}

Herraíz Sarachaga, M. (1997). Conceptos básicos de sismología para ingenieros. Lima: CISMID.

Sadigh, K., Chang, Y., Egan, J. A., Makdisi, F., Youngs, R. (1997). Attenuation Relationships for Shallow Crustal Earthquakes Based on California Strong Motion Data. California.

SENCICO. (2016). Actualización del programa de cómputo orientado a la determinación del peligro sísmico del país. Lima.

Tavera, H., Bernal, I., Condori, C., Ordaz, M., Zevallos, A., \& Ishizawa, O. (2014). Re-Evaluación del Peligro Sísmico Probabilístico. Lima.

Toledo Espinoza, V. (2016). Peligrosidad Sísmica - Evaluación Determinística y Probabilística. Lima: Comunidad para la Ingeniería Civil.

R.N.E. E030. Diseño Sismo resistente. (2018).

\section{Agradecimientos}

Los autores agradecen a la profesional de la escuela Profesional de Ingeniería Civil de la Universidad Privada de Tacna, por su apoyo en la gestión de la investigación. 